\title{
The effects of repetitive transcranial magnetic stimulation in older adults with mild cognitive impairment: a protocol for a randomized, controlled three-arm trial
}

Joy L. Taylor ${ }^{1,2^{*}}$ D, Benjamin C. Hambro', Nicole D. Strossman ${ }^{1}$, Priyanka Bhatt', Beatriz Hernandez ${ }^{1,2}$, J. Wesson Ashford ${ }^{2,3}$, Jauhtai Joseph Cheng ${ }^{1,2}$, Michael IV ${ }^{4}$, Maheen M. Adamson ${ }^{2,5}$, Laura C. Lazzeroni ${ }^{2}$ and Margaret Windy McNerney ${ }^{1,2}$

\begin{abstract}
Background: Mild Cognitive Impairment (MCl) carries a high risk of progression to Alzheimer's disease (AD) dementia. Previous clinical trials testing whether cholinesterase inhibitors can slow the rate of progression from $\mathrm{MCl}$ to AD dementia have yielded disappointing results. However, recent studies of the effects of repetitive transcranial magnetic stimulation (rTMS) in AD have demonstrated improvements in cognitive function. Because few rTMS trials have been conducted in $\mathrm{MCl}$, we designed a trial to test the short-term efficacy of rTMS in $\mathrm{MCl}$. Yet, in both $\mathrm{MCl}$ and $A D$, we know little about what site of stimulation would be ideal for improving cognitive function. Therefore, two cortical sites will be investigated in this trial: (1) the dorsolateral prefrontal cortex (DLPFC), which has been well studied for treatment of major depressive disorder; and (2) the lateral parietal cortex (LPC), a novel site with connectivity to AD-relevant limbic regions.

Methods/design: In this single-site trial, we plan to enroll 99 participants with single or multi-domain amnestic $\mathrm{MCl}$. We will randomize participants to one of three groups: (1) Active DLPFC rTMS; (2) Active LPC rTMS; and (3) Sham rTMS (evenly split between DLPFC and LPC locations). After completing 20 bilateral rTMS treatment sessions, participants will be followed for 6 months to test short-term efficacy and track durability of effects. The primary efficacy measure is the California Verbal Learning Test-II (CVLT-II), assessed 1 week after intervention. Secondary analyses will examine effects of rTMS on other cognitive measures, symptoms of depression, and brain function with respect to the site of stimulation. Finally, selected biomarkers will be analyzed to explore predictors of response and mechanisms of action.

Discussion: The primary aim of this trial is to test the short-term efficacy of rTMS in MCI. Additionally, the project will provide information on the durability of cognitive effects and potentially distinct effects of stimulating DLPFC versus LPC regions. Future efforts would be directed toward better understanding therapeutic mechanisms and optimizing rTMS for treatment of MCl. Ultimately, if rTMS can be utilized to slow the rate of progression to AD dementia, this will be a significant advancement in the field.

(Continued on next page)
\end{abstract}

\footnotetext{
* Correspondence: joyt@stanford.edu

'US Department of Veterans Affairs (VA) Palo Alto Health Care System (151Y),

Sierra-Pacific Mental IIIness Research Education Clinical Center (MIRECC), 3801

Miranda Avenue, Palo Alto, CA 94304-1207, USA

${ }^{2}$ Department of Psychiatry and Behavioral Sciences, Stanford University,

School of Medicine, Stanford, CA, USA

Full list of author information is available at the end of the article
}

(c) The Author(s). 2019 Open Access This article is distributed under the terms of the Creative Commons Attribution 4.0 International License (http://creativecommons.org/licenses/by/4.0/), which permits unrestricted use, distribution, and reproduction in any medium, provided you give appropriate credit to the original author(s) and the source, provide a link to the Creative Commons license, and indicate if changes were made. The Creative Commons Public Domain Dedication waiver (http://creativecommons.org/publicdomain/zero/1.0/) applies to the data made available in this article, unless otherwise stated. 
(Continued from previous page)

Trial registration: Clinical Trials NCT03331796. Registered 6 November 2017, https://clinicaltrials.gov/ct2/show/ NCT03331796. All items from the World Health Organization Trial Registration Data Set are listed in Appendix A.

Protocol version: This report is based on version 1, approved by the DSMB on 30 November, 2017 and amended on 14 August, 2018 and 19 September, 2019.

Keywords: Mild cognitive impairment, Transcranial magnetic stimulation, Prefrontal cortex, Parietal cortex, Neuroimaging, Aging, Alzheimer disease

\section{Introduction}

The goal of this study is to test the efficacy of repetitive Transcranial Magnetic Stimulation (rTMS) as a treatment for amnestic Mild Cognitive Impairment (MCI). MCI describes a clinical entity between healthy cognitive aging and dementia, in which individuals are cognitively impaired but do not meet the full criteria for dementia $[1,2]$. Amnestic MCI (aMCI), in which individuals experience mild memory impairment with or without impairments in other cognitive functions [3-5], can presage dementia due to Alzheimer's Disease (AD), though not every person with aMCI will progress to AD dementia [6]. Among adults aged 65 or older, 16 to $20 \%$ are likely to fit the overall entity of MCI [3, 7]. Once diagnosed, the annual rate of conversion to dementia averages $12 \%$ per year [7, 8]. MCI is an important public health concern due to its prevalence, risk of progression to dementia, and lack of effective treatment.

During the past decade, clinical trials testing whether cholinesterase inhibitors could slow the rate of conversion from MCI to dementia, or at least provide a temporary boost to cognitive performance, yielded disappointing results [9-11]. Reviews of cholinesterase inhibitors, as well as a meta-analysis involving well over $4000 \mathrm{MCI}$ patients, concluded that the low efficacy of cholinesterase inhibitors is outweighed by their adverse effects (e.g. gastrointestinal discomfort, unusual dreams, and leg cramps) [12-15]. A more specific augmentation of the cholinergic system is currently being tested in a 24-month clinical trial of transdermal nicotine (NCT02720445). This clinical trial follows a 6-month pilot trial that demonstrated nicotine improved the primary efficacy measure, attention, in older adults with MCI (standardized mean difference; $\mathrm{SMD}=0.78)$ [16]. To date, no pharmacological treatment has been approved for $\mathrm{MCI}$, and no non-pharmacological treatment has shown satisfactory efficacy for MCI. Systematic reviews of treatments for MCI $[12,13,17]$ indicate that the treatment investigated was ineffective; or if the treatment showed promise of efficacy, the finding still needs to be independently replicated; or the clinical trial was underpowered [12]. Presently, healthy behaviors-particularly exercise, control of cardiovascular risk factors, and cognitive/social activities-are the recommended approaches for coping with MCI. In summary, aside from general health recommendations, there are currently no effective treatments for MCI.

At the leading edge of innovative and safe pilot treatments for improving cognitive function in older adults is rTMS [18, 19], a noninvasive brain stimulation (NIBS) technique. In the Discussion, we outline the therapeutic rationale for rTMS with respect to targeting aMCI abnormalities in brain function. Here, we summarize emerging findings on the efficacy of multiple-session rTMS for improving cognitive function in MCI. Two sham-controlled, blinded trials involving nondemented older adults with MCI have been published [20, 21]. These two trials, which follow up on promising studies showing improvements in cognitive function in patients with mild to moderate Alzheimer's disease (AD) dementia [22-27], employed roughly similar rTMS treatment protocols, in which $10 \mathrm{~Hz}$ high-frequency "excitatory" rTMS [28] was applied over the left dorsolateral prefrontal cortex (DLPFC) for 10 weekday sessions. The first rTMSMCI trial, involving $34 \mathrm{MCI}$ patients, used a parallel groups design [20]. Participants in the active group received $10 \mathrm{~Hz}$ rTMS delivered as 2000 pulses per session (5-s train duration; 25-s inter-train interval (ITI); $110 \%$ MT). Compared to sham, rTMS treatment significantly improved the primary outcome, scores on the Rivermead Everyday Memory Test. This improvement in memory performance was sustained at the 1 month follow-up time point $(\mathrm{SMD}=0.78)$. A second rTMS trial, involving 9 patients with apathy and MCI used a cross-over design [21]. Participants received $10 \mathrm{~Hz}$ rTMS (4-s train duration, 26-s ITI; $120 \% \mathrm{MT}$; 3000 pulses per session) for 10 weekday sessions in the active condition. After a 4-week treatment-free period, the cohorts crossed over and repeated the 10 weekday sessions. Participants showed significant improvement in the primary outcome, the Apathy Evaluation Scale $(p=.045)$, after active treatment compared to sham. Three of the six secondary cognitive outcomes also showed significant improvement; these 
were the Modified and original Mini-Mental State total scores and the Trail Making Test, Part A time score.

Supplementing the scant literature on the efficacy of rTMS for MCI are three additional studies on rTMS for memory improvement: [1] a controlled cross-over trial in "prodromal AD" [29] (i.e., the participants had mild memory loss and were positive for amyloid and tau AD biomarkers [30]) [2]; a single-session rTMS experiment in aMCI [31]; and [3] a single-session rTMS experiment involving older adults with below-normal memory performance [32]. In the single-session experiments, $5 \mathrm{~Hz}$ highfrequency rTMS applied over the left DLPFC for 5 min significantly improved face-name associative memory performance relative to the sham treatment [32], and $1 \mathrm{~Hz}$ low-frequency rTMS (i.e. "inhibitory" rTMS [33]) applied over the right DLPFC for $10 \mathrm{~min}$ following the study of non-verbal stimuli led to enhanced recognition accuracy [31]. In the multisession sham-controlled trial in "prodromal AD" [29], participants $(n=14)$ received $20 \mathrm{~Hz}$ excitatory rTMS (2-s train duration; 28-s ITI; 100\% resting MT; 1600 pulses per session) over the precuneus for 10 weekday sessions in the active rTMS condition. There was a 2-week washout period before crossing over to the other condition. The authors hypothesized that stimulation of the precuneus region of the medial parietal lobe would selectively improve episodic memory. Indeed, participants showed significant improvement in memory, as measured by the Rey Auditory Verbal Learning delayed recall score, following active rTMS; other cognitive scores were not significantly changed [29].

Together, these preliminary findings suggest that rTMS can enhance memory and cognition in MCI patients. Of nine other randomized, controlled rTMS studies in patients with AD dementia [22-27, 3436], eight studies demonstrated significant improvement of cognitive function [22-27, 35, 36]. Yet, we know little about how long the effects of rTMS last, which is key to knowing how frequently rTMS would need to be repeated to slow or prevent progression of impairment. The limited follow-up data published so far come primarily from AD trials, and indicate that five or more sessions of rTMS can produce cognitive benefits lasting up to 4 months [2325]. Moreover, it is not clear what site for noninvasive stimulation is ideal, given the pattern of neurodegeneration and cognitive deficits in MCI and $\mathrm{AD}$ [37]. In the rTMS studies to date on treatment of $\mathrm{MCI}$ and $\mathrm{AD}$, the site most frequently targeted was the DLPFC, the same stimulation site used for treatment of depression. Is the DLPFC truly the ideal stimulation site for MCI and AD? Stimulation of the LPC gained proof of concept in a study of healthy young adults, in which 5 daily sessions of rTMS over the LPC led to significant improvement of associative memory [38]. Ultimately, stimulating both prefrontal and parietal regions might provide superior clinical outcomes given the profound effects of $\mathrm{AD}$ on widespread interconnected brain regions $[39,40]$. Clearly, a greater understanding of how rTMS applied over a cortical site leads to changes in cognitive and brain function is important in deciding whether one site is ideal and sufficient, or whether stimulating multiple sites could be superior [41]. The current protocol is designed to rigorously test the short-term efficacy of rTMS in aMCI, while also commencing to address crucial questions about choice of stimulation site and durability of effects.

\section{Study aims}

The primary aim is to test the hypothesis that patients receiving active rTMS will show more improvement in memory at the 1-week post-intervention assessment than the sham-control group. Secondarily, we aim to:

1) Assess the durability of rTMS effects on memory over a 6-month follow-up period, which is longer than assessed in previous studies;

2) Examine effects of rTMS on behavior and brain function related to the site of stimulation, which is a novel approach in the AD spectrum;

3) Explore patient characteristics that could be useful in identifying who responds preferentially to rTMS or to a particular stimulation site.

\section{Methods/design \\ Study design and overview}

Our hypotheses regarding the effects of rTMS on memory in persons with aMCI will be tested in a randomized, 2-stimulation-site, sham-controlled, 3arm double-blind design, in which bilateral rTMS is delivered as: [1] active rTMS of the DLPFC, [2] active rTMS of the Lateral Parietal cortex (LPC), or [3] sham/inactive rTMS (evenly split between each coil location). Participants will receive 20 treatment sessions. Behavioral measures will be collected at baseline, at 1-week post-intervention, and at two subsequent follow-up visits at 3 and 6 months. In addition to the primary efficacy measure of memory, secondary measures of global cognitive function, language, executive control, depressive symptoms, and everyday function will be obtained. Resting-state functional Magnetic Resonance Imaging (rs-fMRI) 
scans will be acquired at baseline and 1-week postintervention to examine effects of rTMS on functional connectivity. The rTMS-MCI project is designed to ultimately include data from 99 aMCI participants ( $n=33$ per group), with measures of cognitive efficacy, brain function, and predictors of response to rTMS. The study site is the Stanford/VA Aging Clinical Research Center, an outpatient clinical research center located at the VA Palo Alto Health Care System near the Stanford University School of Medicine. The trial design and study flow are shown graphically in Fig. 1.

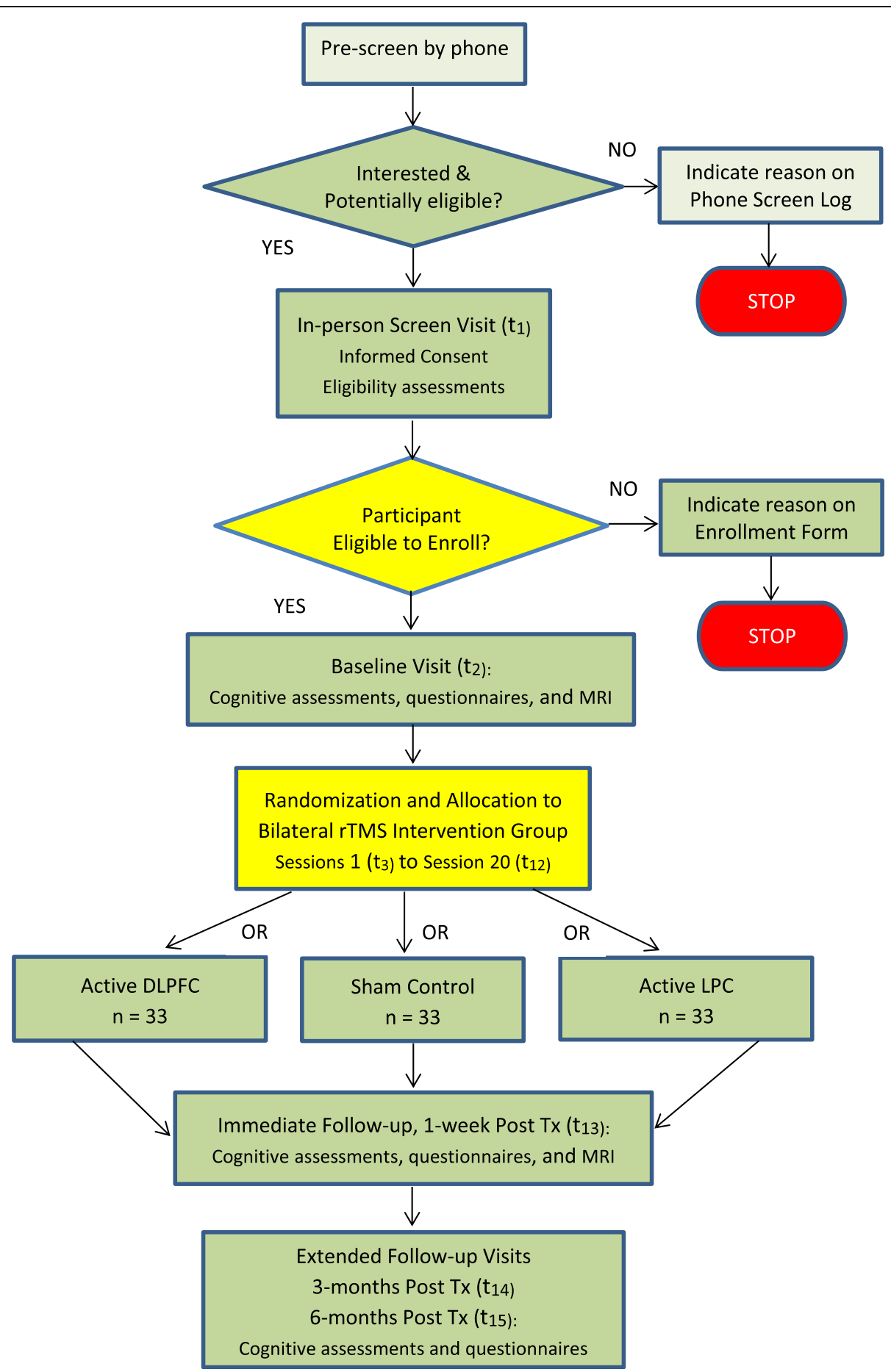

Fig. 1 A summary of the study design and participant flow through the study 


\section{Participants}

The target population is individuals with single- or multi-domain amnestic MCI (aMCI). For this study, we use the Alzheimer's Disease Neuroimaging Initiative (ADNI-3) characterization of aMCI, which uses a combination of clinical and neuropsychological assessments to classify participants into categories of normal cognition, MCI, or AD dementia [42].

\section{Enrollment}

\section{Inclusion criteria}

Participants are eligible for randomization if they fulfill the following inclusion criteria:

1. Age 55-90 years inclusive;

2. Diagnosed with aMCI as defined by ADNI-3 [42];

a. Participant must express a subjective memory concern as reported by participant or recalled by study partner or clinician.

b. Below normal memory function documented by scoring below the education-adjusted cutoffs on the Logical Memory II subscale (Delayed Paragraph Recall, Story A) from the Wechsler Memory Scale-Revised (the maximum score is 25): a) less than 11 for 16 or more years of education; b) less than or equal to 9 for $8-15$ years of education; c) less than or equal to 6 for $0-7$ years of education.

c. Mini-Mental State Exam score between 24 and 30 (inclusive). (Exceptions may be made for subjects with less than 8 years of education at the discretion of the project director).

d. Clinical Dementia Rating $=0.5$. Memory Box score must be at least 0.5 .

e. General cognition and functional performance sufficiently preserved such that a diagnosis of dementia cannot be made by the physician at the time of the screening visit.

3. Stable medications (including any dementiarelated meds) for at least 4 weeks prior to the Baseline visit;

4. Geriatric Depression Scale score less than 6;

5. Ability to obtain a motor threshold, determined during the screening process;

6. Study partner available; living situation enables attendance at clinic visits;

7. Visual and auditory acuity adequate for neuropsychological testing;

8. Good general health with no diseases expected to interfere with the study, as determined by the referring Memory Clinic Physician or the rTMS Study Physician;
9. Participant is not pregnant or of childbearing potential;

10. Modified Hachinski Ischemic score less than or equal to 4 ;

11. Agree to DNA extraction for single nucleotide polymorphism (SNP) genotyping;

12. Able to understand study procedures and comply with them for the entire length of the study.

\section{Exclusion criteria}

1. Prior exposure to rTMS within the past 12 months;

2. Magnetic field safety concern such as a cardiac pacemaker, cochlear implant, implanted device in the brain, or metal fragments or foreign objects in the eyes, skin or body;

3. Any significant neurological disease other than suspected incipient Alzheimer's disease;

4. Unstable cardiac disease or recent $(<3$ months previous) myocardial infarction. Any significant systemic illness or unstable medical condition that could lead to difficulty with protocol adherence;

5. History of epilepsy or repetitive seizures, as determined by patient report or chart review;

6. History of a medical condition or current use/abuse of medications and substances that increase the risk of a seizure, specifically:

a. Traumatic brain injury within the past 2 months;

b. Unable to safely withdraw, at least 4 weeks prior to Baseline, from medications that substantially increase the risk of having seizures (for example, theophylline, clozapine, and methylphenidate; the complete list of Exclusionary Medications is available from the corresponding author).

c. Current or past history of a mass lesion, cerebral infarct, or other non-cognitive active neurological disease that would increase the risk for seizure.

d. Stimulant abuse within the previous 90 days.

Cocaine and abuse of amphetamine and methylphenidate are associated with an increased risk of seizures;

e. Major depression or bipolar disorder (DSM-IV) within the past 1 year, or psychotic features within the last 3 months that could lead to difficulty with protocol adherence;

7. Taking sedative hypnotics or medications with anticholinergic properties and unable to withdraw at least 4 weeks prior to Baseline;

8. Current alcohol or substance abuse (not including caffeine or nicotine) within the past 1 year, as 
determined by chart review, participant or study partner report, or greater than "moderate" alcohol use defined by the Quantity-Frequency-Variability Index [43];

9. Any contraindications for MRI studies (e.g. severe claustrophobia, pregnancy, pacemaker, weight above $160 \mathrm{Kg}$ maximum allowed by MRI scanner);

10. Participation in another concurrent clinical trial;

11. Inability or unwillingness of individual or legal representative to give written informed consent.

\section{Recruitment strategies}

Multiple strategies will be used to achieve adequate enrollment and reach the target sample size, including the following: (1) targeted recruitment via memory clinics that are affiliated with the Stanford University School of Medicine; (2) collaboration with relevant online research registries such as the Alzheimer's Prevention Registry and the Brain Health Registry; and (3) flyer postings at the VA Palo Alto Health Care System's facilities; (4) networking with local neurologists, psychiatrists and neuropsychologists; and (5) direct-mail advertising to individuals based on age and geography.

\section{Determination of study eligibility}

As illustrated in in Fig. 1, determination of study eligibility is a two-stage process. First, interested individuals are pre-screened by telephone using an IRB-approved screening script. Potentially eligible participants are then invited to come to the clinic for a Screening Visit $\left(t_{1}\right)$ that begins with obtaining written informed consent. Next, eligibility assessments are performed, as described in the "Measures" section. If the participant meets all eligibility criteria, behavioral measures and an MRI are acquired at a separate Baseline Visit $\left(t_{2}\right)$. Following completion of the Baseline procedures, the Database Manager randomizes the participant to one of the three intervention arms. More details on randomization and double-blinding are described under "Randomization." Briefly, a unique 6-digit number is assigned to the participant; when this 6digit number is entered into the interface of the TMS device, the software enables the device and an unmarked active-placebo $(\mathrm{A} / \mathrm{P})$ coil to deliver the appropriate intervention (active or sham) to that participant.

\section{Intervention}

rTMS stimulation protocol, parameters, and coil placement The intervention is administered as 20 weekday sessions during a period of 2 to 4 weeks. Typically, a participant will have a morning session and an afternoon session. The intervention uses rTMS stimulation procedures that have proven evidence of efficacy to improve cognitive function in AD dementia, based on two meta-analyses [44, 45]. Specifically, high-frequency $(10 \mathrm{~Hz})$ rTMS is delivered bilaterally, as this procedure was significantly effective in improving the cognition of AD patients [44]. Stimulation will be applied over the right hemisphere first, and immediately followed by the left hemisphere, following the procedure of two previous bilateral stimulation trials [23, 24]. Coil placement is guided by a Localite neuronavigation system (Localite $\mathrm{GmbH}$, Bonn, Germany) using the MRI data acquired at the baseline visit. By using neuronavigation, we aim to optimize subjects' clinical responses, irrespective of whether the subject is assigned to DLPFC or LPC stimulation. DLPFC participants will be stimulated on the left at the MNI coordinate $\mathrm{x}=$ $-38, y=44, z=26$, which was identified by Fox et al. [46] as a DLPFC site associated with more efficacious outcomes in rTMS treatment of depression. LPC participants will be stimulated on the left at the MNI coordinate $\mathrm{x}=-47, \mathrm{y}=-68, \mathrm{z}=36$, which was the MNI centroid used by Wang et al. [38] for fMRI-guided neuronavigation of rTMS; Wang et al. [38] successfully modulated cortical-hippocampal connectivity and improved associative memory performance in healthy, young adults. For both DLPFC and LPC participants, stimulation of the right hemisphere is targeted to the corresponding coordinate on the right (e.g. $x=47, y=-68, z=36$ to target the right LPC).

The same stimulation parameters are used to deliver both of the active interventions (DLPFC and LPC); specifically, a pulse frequency of $10 \mathrm{~Hz}$ with a 4-s train duration (40 pulses) and 11-s ITI. During each session, 2000 pulses will be applied over each hemisphere at $120 \%$ of the participant's resting motor threshold, which is individually measured for each hemisphere. In summary, this $10 \mathrm{~Hz}$ stimulation protocol delivers 4000 pulses per session and up to 8000 pulses per day, with a total of 80,000 pulses over 2- to 4-week period.

\section{Equipment and masking procedures}

The intervention is delivered using a MagPro X100 magnetic stimulator. The Cool-B65-A/P coil system creates auditory "clicks" for sham intervention that match the click sounds of active rTMS. Additionally, for each session, whether sham or active, each participant wears scalp electrodes through which a lowvoltage, low electric current $(2-6 \mathrm{~mA}$ at no more than $100 \mathrm{~V}$ ) is passed in order to provide cutaneous 
stimulation that mimics the sensation of actual rTMS. Additional details are provided in $\mathrm{Mi}$ et al. [47] and the TMS operator manual developed for this protocol. Note: Additional file 1: "List of supplementary documents" provides a list of rTMS manuals that can be obtained from the authors.

\section{Intervention discontinuation}

The study intervention will be discontinued for a participant if:

1. The participant has a seizure;

2. There are signs or reports of inability to tolerate the intervention, or if the study physician feels it is in the participant's best interest to discontinue with the intervention;

3. Or, there is any other health or safety concern that contraindicates continuation based on the judgment of the study physician.

Such participants will be encouraged to return for post-intervention follow-ups. Except for the MRI scan, all other outcome measures, adverse effect (AE) information and safety assessments would be collected during the follow-up period.

\section{Measures}

Additional file 2 Table S1 lists the schedule of all study assessments, including eligibility, safety, and outcome measures. The assessments are described briefly below:

\section{Eligibility assessments}

\section{Assessment of aMCI}

During the screening visit, the study coordinator assesses memory and global cognitive function using the Logical Memory (Story A) portion of the Wechsler Memory Scale-Revised [48] and the Mini-mental Status Exam (MMSE) [49]. Independently of the study coordinator, a certified rater assesses the participant's functional competence using the Clinical Dementia Rating scale (CDR) [50]. To screen for depression and ensure that a mood disorder is not a primary cause of memory impairment, the participant completes the Geriatric Depression Scale (GDS) [51]. To confirm the presence of subjective memory decline, the participants fills out the Cognitive Change Index questionnaire [52]. A physician interviews the participant and provides a modified Hachinski rating [53] to ensure that cerebrovascular disease is not a primary cause of memory impairment. The classification criteria for aMCI that are used in this clinical trial are listed above under "Inclusion criteria."
Safety, blinding, and TMS acceptability assessments TMS/MRI safety assessments

During the screening phase and prior to rTMS or MRI sessions, participants answer questionnaires regarding their safety in undergoing rTMS intervention or an MRI (surgical history, epilepsy history, presence of metal or cardiac pacemaker, etc.). Participants also undergo a detailed history, physical, and neurological screening exam by a licensed physician whose approval must be given before enrollment. Vital signs as well as medication, sleep, and substance use history are measured throughout the study to ensure participant safety.

Collection and monitoring of any AEs begins at the time the participant signs the informed consent form and continues throughout the 6-month postintervention follow-up phase. Specific procedures for collecting, assessing, reporting, and managing solicited and spontaneously reported are described in the detailed study protocol, which is available from the authors. (See Additional file 1: "List of supplementary documents.") The PI and research team will notify the Data and Safety Monitoring Board (DSMB) and the NIA Program Official of all Serious Adverse Events within $24 \mathrm{~h}$ of study staff's knowledge of the event. All AEs will be reported annually or more often to the DSMB, Stanford IRB, and NIA staff. Oversight by the DSMB is described further in the "Monitoring" section of the Methods.

\section{Outcome measures}

\section{TMS blinding and acceptability questionnaires}

Before receiving and after completing the intervention, both the participant and the TMS operator are asked to guess the participant's group assignment. At the end of intervention, the participant answers openended questions about treatment satisfaction and the tolerability of rTMS.

\section{Outcome measures \\ Primary outcome measure}

The primary outcome is change in memory performance, observed 1 week following intervention $\left(t_{13}\right)$. Memory is assessed using the California Verbal Learning Test (CVLT-II) [54]; the primary dependent variable is the CVLT-II Total score summed over Trials 1-5 (possible range 0-80; higher values represent a better outcome). The CVLT is a two-list memory task that assesses multiple aspects of verbal learning and episodic memory. List A of the CVLTII contains 16 concrete words; four words each from four categories (animals, vegetables, ways of traveling, and furniture). List A is presented for 5 learning trials. After presentation and recall of another List 
(B), a short-delay free recall and cued recall of list A is performed. After a 20-min delay filled with nonverbal testing, long-delay free recall and cued recall are assessed, followed by a yes/no recognition test for List A.

Use of the CVLT-II Total (T1-5) score as the primary outcome should allow for a wide range of scores that are neither at floor nor at ceiling level, based on previous research [55-57]. The CVLT-II Total 1-5 score also has high diagnostic validity. The CVLT-II Total 1-5 score had the highest predictive value for MCI-to-dementia conversion [58] and was the best variable for distinguishing MCI from normal cognitive function [59], in comparison to other measures of episodic memory. Additional advantages of the CVLT-II include: excellent retest reliability (e.g. Total 1-5 reliability: $\mathrm{r}=.82$ ); the availability of an alternate test form (Total 1-5 alternate-form reliability: $\mathrm{r}=.79$ ), and relevant normative data. In summary, we selected the CVLT-II for reasons related to sensitivity, validity, reliability, and, as discussed in the next section, its potential utility for detecting treatment-response differences related to the cortical site of rTMS stimulation.

\section{Secondary behavioral outcome measures}

A variety of secondary measures are being collected to compare and contrast the effects of DLPFC and LPC stimulation. First of all, the clustering and recall scores of the CVLT-II will provide key measures of memory encoding and retrieval, which in turn relate to the distinct roles that DLPFC and LPC regions have in supporting these processes [60]. The other secondary outcome measures were selected on the basis of previous studies of rTMS in $\mathrm{MCI}$ and $\mathrm{AD}$ that reported statistically significant improvements in global cognitive function [22-24, 61, 62], language [25-27], instrumental activities of daily living [23], and a reduction of depressive symptoms [23]. The following secondary behavioral measures are collected at 4 timepoints: baseline $\left(t_{2}\right)$, 1-week post intervention $\left(t_{13}\right)$, 3-months post intervention $\left(t_{14}\right)$; and at 6months post intervention $\left(t_{15}\right)$ :

\section{Cognitive measures}

1. Secondary CVLT-II [54] verbal episodic memory measures of interest are: semantic clustering (chance-adjusted) Trials 1-5; and the short- and long-delay free recall scores $(0-16$; higher values represent a better outcome). Our working hypothesis is that DLPFC stimulation could result in a higher level of semantic clustering compared to LPC stimulation. By contrast, LPC stimulation could result in improved recall, assessed by the CVLT-II short- and long-delayed recall scores.

2. Global cognitive function is measured using the Montreal Cognitive Assessment (MoCA) [63] Total score (0 to 30; higher values represent a better outcome).

3. Visuospatial episodic memory is measured using the Brief Visuospatial Memory Test-Revised (BVMTR) [64], Trials 1-3 Total raw score (0-36; higher values represent a better outcome).

4. Language abilities are measured using: Category Fluency [65], total number of correct responses in $60 \mathrm{~s}$ (higher values represent a better outcome); and 42-item Boston Naming Test (BNT) [66], Total score (0-42; higher values represent a better outcome).

5. Visuoconstructional function is measured using the Rey-Osterrieth Complex Fig [67, 68]., Copy score (0-36; higher values represent a better outcome).

6. Speed of processing and executive control are measured by the Trail Making Test, Parts A and B (number of seconds to complete; lower values represent a better outcome) $[69,70]$.

\section{Functional questionnaires}

The participant and study partner will be asked to independently fill out the Everyday Cognition Scale [71], which asks about perceived changes in performance of everyday tasks (ECog Total score, range: 39156; higher values represent a worse outcome). The study partner will also complete the Functional Activities Questionnaire (FAQ) [72], which assesses the participant's level of independence in performing everyday activities (FAQ Total score, range: 0-30; higher values represent a worse outcome).

\section{Depressive symptoms}

The participant will complete the 15-item GDS [51]. (Total score, range: 0-15; higher values represent a worse outcome). Because the GDS is also an eligibility assessment, it is obtained prior to baseline at $t_{1}$; the $t_{1}$ value will be treated as the baseline timepoint.

\section{Secondary neuroimaging outcomes}

MRI scans are acquired at baseline $\left(t_{2}\right)$ and 1-week post intervention $\left(t_{13}\right)$ on a 3 Tesla scanner (GE Healthcare, Chicago, Illinois). During each $40 \mathrm{~min}$ scan, the following five sequences are acquired: anatomical 3D T1-weighted gradient echo sequence [sagittal T1-weighted inversion-recovery-prepared, fast spoiled gradient recalled BRAin VOlume (BRAVO) imaging, $1 \mathrm{~mm}^{3}$ voxel size], rs-fMRI [ $3 \mathrm{~mm}^{3}$ voxel size, 9 min scan duration, using simultaneous multi- 
slice (SMS) acquisition]; 3D T2-weighted FLAIR (fluid-attenuated inversion recovery) sequence (sagittal imaging combined with an isotropic 3D fast spin echo acquisition, "CUBE"); axial non-contrast Arterial Spin Labeling (ASL) perfusion imaging; and diffusion-weighted echo-planar imaging (DW-EPI and epi2alt acquisitions, axial 2D, 25 directions for each acquisition). Functional connectivity metrics will be derived from the rs-fMRI scans for use in secondary and exploratory analyses.

\section{Secondary biomarker variables}

Plasma levels of brain-derived neurotropic factor (BDNF) are measured from fasting blood samples that are collected at the first $t_{3}$ and last $t_{12}$ morning intervention sessions to gain information about mechanisms of action of rTMS with respect to brain plasticity. Genomic DNA is acquired from blood at $t_{3}$ to explore genetic predictors of response to rTMS. Additional file 4 describes procedures for the collection, processing, and storage of biological specimens.

\section{Randomization}

Participants are randomized in a 2:1 ratio to active rTMS or sham at baseline. Within the active and sham groups, participants are randomized in a 1:1 ratio to the two unmasked cortical sites. The method of random assignment to treatment (active vs. sham) and to cortical site (DLPFC vs LPC) is determined by a computer-generated random sequence. An adaptive randomization scheme is being used so that equal numbers of participants are randomized to each of the 3 treatment groups within blocks of every 6 participants. For each block, 4 active and 2 sham treatment numbers are randomly assigned. To ensure proper allocation of participants, a block of 6 participants consists of: 2 active DLPFC, 2 active LPC, 1 sham DLPFC and 1 sham LPC.

\section{Blinding / masking}

To accomplish double-blinding, the study's database manager randomizes each participant to treatment and maintains the list of randomization codes in an encrypted restricted-access file; only the Database Manager knows the encryption key. Thus, study staff-including the principal investigator, study coordinator, and TMS operator-and participants are blinded to group assignment.

\section{Statistical analysis plan and sample size calculations}

Outcome measures will be analyzed at study completion by the database manager under the supervision of a Ph.D. biostatistician. For the purposes of analyses, there will be 3 equally sized treatment groups of interest: 1) active DLPFC; 2) active LPC; and 3) sham. The sham group is comprised of all subjects assigned to the sham group, regardless of the position of the sham coil.

\section{Primary analysis}

The primary hypothesis is that participants receiving active rTMS will show more improvement in memory than the sham group at the 1-week post-intervention assessment. An Intention-to-treat (ITT), Analysis of Covariance (ANCOVA) will be run on the CVLT-II Trials 1-5 Total raw $t_{13}$ score using the baseline CVLT-II score as a covariate. ITT includes all randomized patients who started at least 1 intervention session. Testing the primary hypothesis will involve two statistical tests: Test 1 will compare active DLPFC to sham; Test 2 will compare active LPC to sham. The two tests will be conducted at a 2-sided alpha level of 0.05 , adjusted for two tests.

Statistical power We powered the study to detect a moderately large effect size (Cohen's $d=0.80$ ) of either rTMS treatment on the primary outcome. Moderately large effect sizes of $1.0,0.80$, and 0.80 were reported in two meta-analyses of rTMS studies in AD [44] [45] and one rTMS trial in MCI (which used unilateral rTMS [20]. With a total sample size of 99 participants, our study is estimated to have $80 \%$ power with a multiple testing penalty for two tests. For follow-up analyses of differences related to the site of stimulation, this sample size will provide $80 \%$ power to detect a large difference $(\mathrm{d}=1.00)$. For all analyses, we will convey practical significance by reporting effect sizes and confidence intervals, in addition to reporting statistical significance.

\section{Secondary analyses}

For all analyses, parameter estimates will be reported for Model 1: active DLPFC vs. sham, and for Model 2: active LPC vs. sham.

Durability The durability of rTMS effects on memory over the entire study period will be examined using mixed-effects growth curve modeling [73]. The magnitude of treatment effects at the 3-mos and 6mos time points will be reported. Our working hypothesis is that an effect of rTMS on the primary outcome, the CVLT-II Trials 1-5 Total score, will be sustained up to 3 months.

Behavioral differences related to the site of brain stimulation The effect of rTMS effects on the secondary CVLT-II memory scores and other cognitive outcomes, on depressive symptoms, and on 
functioning will be examined using mixed-effects growth curve modeling [73]. Separate models will be fit for each secondary outcome. To aid in contrasting the magnitude of effects of DLPFC vs. LPC stimulation, parameter estimates and 95\% confidence intervals will be reported for each stimulation site. Potential differences in the magnitude of effects on semantic clustering and delayed recall sub-scores of the CVLT-II will be particular interest.

Functional connectivity With respect to therapeutic effects of rTMS on functional connectivity, changes in connectivity within the posterior Default-mode network (DMN), and changes between the DMN and networks that support task engagement [74, 75] are of particular interest. This is because of reports that aMCI patients, compared to cognitively unimpaired older adults, show 1) abnormal connectivity within the DMN, as well as 2) abnormal interactions between the DMN and other networks, in particular the Salience network and the Central Executive network (CEN) [76]. To examine effects of rTMS on functional connectivity, two sets of analyses will be performed on the baseline $\left(t_{2}\right)$ and 1-week post intervention $\left(t_{13}\right)$ rs-fMRI data: [1] rTMS effects on connectivity within the DMN. The aim of these analyses is to discover the extent to which rTMS restores connectivity of the posterior cingulate cortex (PCC) within regions of the DMN; and [2] rTMS effects on between-network connectivity. We will examine if rTMS reduces abnormally strong functional connectivity between the CEN and DMN, i.e. makes the two networks more anti-correlated. Change in connectivity from baseline to 1-week post intervention will be computed, and contrasts will be made between each active rTMS group and the sham group, and between the LPC and DLPFC groups. Appropriate corrections for multiple comparisons will be made to provide an overall alpha probability of a type I error of $<0.05$.

\section{Exploratory analyses}

Change in BDNF levels from the beginning to the end of intervention will be examined using ANCOVA. Our working hypothesis is that BDNF levels will increase more in either active rTMS group, compared to that in the sham group. Though a direct link between rTMS and synaptic plasticity remains to be demonstrated in humans, an effect of rTMS on BDNF levels would be consistent with a beneficial effect on brain plasticity. Finally, baseline functional connectivity, genetic, and selected clinicodemographic variables will be explored as potential moderators of response to rTMS. The results of these exploratory analyses are useful toward generating hypotheses directed toward ultimately identifying who responds preferentially to rTMS or to a particular stimulation site.

\section{Dissemination of research results}

Following completion of the study, a manuscript will be prepared for the primary outcome (change in CVLT-II performance observed 1 week following intervention) and for the secondary memory-related outcomes, such as the durability of effects of rTMS on memory over the study period. Additional manuscripts may be prepared to report on the effects of rTMS on other secondary behavioral outcomes (cognitive function and mood), and secondary neuroimaging and biomarker variables. Every manuscript will be reviewed and approved by the protocol director, key personnel, database manager, and all other co-authors prior to submission. Each participant will receive a summary of the study results along with revelation of their respective treatment group.

\section{Monitoring \\ Data safety monitoring board (DSMB)}

An independent, external DSMB has been assembled to monitor participant safety, the progress of the study, and the quality of data collection. Each DSMB member-3 psychiatrists and 1 biostatistician-is an expert in the area of TMS, clinical trials, and/or MCI. The DSMB meets twice annually, typically by teleconference call. The DSMB determines when they should be un-blinded to treatment assignment for the reviewing of AEs. The DSMB advises the PI and funder whether the study should continue or be stopped. The DMSB or funder will determine if a planned interim analysis should be conducted. All protocol modifications are first approved by the DSMB, and then submitted to the IRB for its approval. An modification that affects participant activities or risk-to-benefit ratio will be incorporated into the informed consent form documents and communicated to participants. The DSMB will discharge itself from its duties when the last participant completes the study.

\section{Discussion}

There is increasing evidence from clinical trials that rTMS can improve cognitive function in older adults with MCI [20, 21, 31], prodromal AD [29] and probable AD dementia [22-27, 35, 36]. These trials involved relatively small sample sizes and limited follow-up after intervention. Some studies did not have the benefit of a convincing sham coil system. The current protocol is designed to achieve rigorous, 
reproducible methods to test the efficacy of rTMS to improve memory in aMCI, while commencing to address essential questions of the duration of therapeutic effects and selection of stimulation site.

To our knowledge, this would be the first study to investigate differences between the effects of rTMS applied over a DLPFC versus an LPC site within the same study design. Stimulating the DLPFC could have effects that would not be achieved with LPC stimulation, such that stimulating the DLPFC may differentially improve executive function, encoding of new information, and depressive symptoms. Conversely, stimulating the LPC may differentially improve memory retrieval and retention-the core cognitive domain of aMCI.

This would be the first clinical trial of rTMS in aMCI to measure changes in brain function, as assessed with pre-post rs-fMRI and functional connectivity metrics. It is believed that rTMS offers a means to modulate the brain's large-scale networks $[77,78]$ such as the DMN [79-81] and fronto-parietal /CEN [82] networks by positioning the rTMS coil over a cortical node of that network [83]. The effects of targeted stimulation are measurable weeks later in distal regions that are directly or indirectly connected with the cortical site stimulated [84]. Thus, precisely guided rTMS has the potential to improve brain network dynamics that are selectively abnormal in an individual patient or certain condition [85].

For patients with aMCI, abnormal connectivity involving the DMN is a therapeutic target for rTMS. Network nodes that are accessible include parietal cortical sites, which in turn show connectivity to other DMN regions in the limbic, medial temporal, and inferior temporal cortices. In aMCI, the PCC of the medial limbic lobe most consistently shows reduced resting-state activity [86]. The PCC is a key node within the DMN [87-89] and a cortical hub that dynamically participates in interactions of the DMN with other brain networks $[88,90]$. An rTMSfMRI study in healthy young adults showed that multiple sessions of rTMS, when applied to a site over the LPC, increased connectivity between the LPC and PCC, the LPC and hippocampus, and other regions within the posterior DMN [38]. Thus, stimulating the LPC of aMCI patients has the potential to help restore loss of functional connectivity within the posterior DMN and help restore the PCC's connectivity with other DMN regions in the inferior temporal and medial temporal cortices (in particular, parahippocampal and fusiform gyri and the hippocampi).

On the other hand, rTMS delivered to the right or left DLPFC region can potentially improve network interactions between the CEN and DMN regions, based on rTMS studies involving adults with major depressive disorder [91] and healthy young adults [83]. Importantly, rs-fMRI studies of aging and the AD spectrum have revealed altered network interactions between the DMN and networks such as the CEN, which in turn correlate with lower cognitive performance $[40,92-94]$. Thus, stimulating the DLPFC using rTMS has potential to improve DMN: CEN interactions in older adults with MCI. In summary, the present clinical trial aims to gain novel insights as to how rTMS could improve brain network function in aMCI patients by investigating changes in functional connectivity after noninvasive cortical stimulation.

If this clinical trial obtains a signal of improved memory performance or favorable changes in functional connectivity, the next step in the clinical development of rTMS for aMCI would be a multi-site Phase III trial with the inclusion of AD biomarkers. For individuals who are in the Alzheimer's spectrum [95], the progression of $\mathrm{AD}$ from $\mathrm{MCI}$ to dementia reflects a cascade of neurodegenerative processes, of which amyloid- $\beta$ facilitated tauopathy is a leading account of $\mathrm{AD}$ progression [96-98]. If it could be shown that neuromodulation via noninvasive brain stimulation could actually move an AD biomarker of pathologic $A \beta$, tau, or neuronal status in a positive direction, this would be a major step forward in developing disease-modifying treatments for AD.

The prediction of responsivity to noninvasive brain stimulation is a parallel goal of future clinical development. There is evidence that individuals with mild dementia show a better cognitive response to rTMS than do those with more severe dementia [23, 36]. The pattern of differential response to rTMS in relation to dementia severity is plausibly due to decline of inherent neuroplasticity, greater skull-to-cortex distance, or other factors. Conceivably, indices of baseline functional connectivity, genetic variants (e.g. BDNF and Apolipoprotein $\mathrm{E}$ (APOE), or more easily measured, low-cost clinico-demographic variables will ultimately help predict response to rTMS and other modes of noninvasive brain stimulation. There are currently no effective treatments for MCI, aside from general health recommendations. If rTMS can be effectively utilized in older adults with MCI to delay progression to dementia, this would represent a significant advance in the field of $\mathrm{AD}$ and related disorders.

\section{Study status}

At the time of submission, the study has enrolled 24 study participants and has not completed participant recruitment or data collection. 


\section{Appendix}

Table 1 World Health Organization (WHO) trial registration data set

\begin{tabular}{|c|c|}
\hline Data Category & Information \\
\hline $\begin{array}{l}\text { Primary registry and trial } \\
\text { identifying number }\end{array}$ & ClinicalTrials.gov NCT03331796 \\
\hline $\begin{array}{l}\text { Date of registration in } \\
\text { primary registry }\end{array}$ & 6 November, 2017 \\
\hline Source of monetary support & The National Institute on Aging \\
\hline Primary Sponsor & The National Institute on Aging \\
\hline Contact for public queries & $\begin{array}{l}\text { Joshua Teso (Study Coordinator) } \\
\text { 650-852-3457 } \\
\text { Joshua.Teso@va.gov }\end{array}$ \\
\hline Contact for scientific queries & $\begin{array}{l}\text { Joy Taylor, PhD } \\
\text { joyt@stanford.edu } \\
\text { Stanford/NA Aging Clinical Research Center } \\
3801 \text { Miranda Avenue (151Y) } \\
\text { VA Palo Alto Health Care System } \\
\text { Palo Alto, CA, USA 94304-1207 }\end{array}$ \\
\hline Public Title & Noninvasive Brain Stimulation for Mild Cognitive Impairment \\
\hline Scientific title & Noninvasive Cortical Stimulation to Improve Memory in Mild Cognitive Impairment \\
\hline Countries of recruitment & USA \\
\hline $\begin{array}{l}\text { Health condition or } \\
\text { problem studied }\end{array}$ & $\begin{array}{l}\text { Mild Cognitive Impairment }(\mathrm{MCl}) \text {, } \\
\text { Other terms: Mild Neurocognitive Disorder, Memory Decline, Memory Loss, Memory Impairment }\end{array}$ \\
\hline Intervention (s) & $\begin{array}{l}\text { Repetitive transcranial magnetic stimulation (rTMS), } 10 \mathrm{~Hz}, 20 \text { sessions Active comparators (Arms } 1 \text { and 2): } \\
\text { rTMS Bilateral Dorsolateral Prefrontal Cortex (DLPFC); rTMS Bilateral Lateral Parietal Cortex (LPC) } \\
\text { Placebo comparator (Arm 3): } \\
\text { Inactive sham coil treatment }\end{array}$ \\
\hline $\begin{array}{l}\text { Key Inclusion and Exclusion } \\
\text { criteria }\end{array}$ & $\begin{array}{l}\text { Inclusion: } \\
\text { Age 55-90, amnestic MCl, stable medications, Geriatric Depression Scale score less than 6; Ability to obtain a motor } \\
\text { threshold; Study partner available; Visual and auditory acuity adequate for neuropsychological testing; Good general } \\
\text { health with no diseases expected to interfere with the study. } \\
\text { Exclusion: } \\
\text { Magnetic field safety concern such as a cardiac pacemaker, cochlear implant, metal fragments in the eyes, skin or body, } \\
\text { or pregnancy; Any significant neurological disease other than suspected incipient Alzheimer's disease; Unstable cardiac } \\
\text { disease or recent myocardial infarction; Any significant systemic illness or unstable medical condition that could lead } \\
\text { to difficulty with protocol adherence; History of epilepsy or repetitive seizure; History of a medical condition or current } \\
\text { use/abuse of medications and substances that increase the risk of a seizure (e.g. recent traumatic brain injury, current } \\
\text { use of stimulants), Known history of major depression or bipolar disorder within the past year; Current alcohol or } \\
\text { substance abuse (not including caffeine or nicotine) within the past year. }\end{array}$ \\
\hline
\end{tabular}

Study type

Interventional Device / Phase II

Allocation: randomized

Intervention model: parallel assignment (3 arms)

Masking: double blind

Primary Purpose: treatment

Date of first enrollment

16 May 2018

Target sample size

99

Recruitment status

Recruiting

Primary outcomes

Change in memory, as measured by the California Verbal Learning Test-II (CVLT-II) Trials 1-5 Total raw score [Time Frame: Baseline, 1 week after completing the 20-session intervention]

Key Secondary outcomes

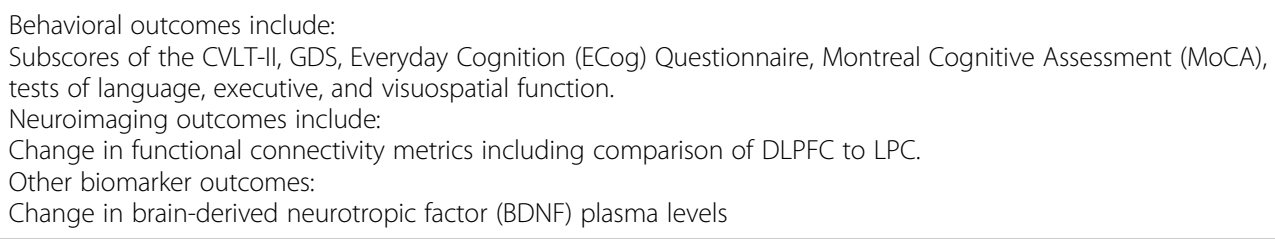




\section{Supplementary information}

Supplementary information accompanies this paper at https://doi.org/10. 1186/s12883-019-1552-7.

Additional file 1. List of supplementary documents. The detailed study protocol (which includes details of data collection, AE monitoring, statistical considerations, and data management), exclusionary medications, and two rTMS manuals are available from the authors.

Additional file 2: Table S1. Schedule of enrollment, interventions, and assessments.

Additional file 3. Informed consent documents. Research consent forms for the clinical trial and for an ancillary study involving urine specimens.

Additional file 4. Biological specimens manual. Appendix of procedures for the collection, processing, and storage of biological specimens for the current trial.

\section{Abbreviations}

A/P: Active-placebo; AD: Alzheimer's Disease; ADNI: Alzheimer's Disease Neuroimaging Initiative; AE: Adverse Event; aMCl: Amnestic Mild Cognitive Impairment; ANCOVA: Analysis of Covariance; APOE: Apolipoprotein E; ASL: Arterial Spin Labeling; BDNF: Brain-derived Neurotropic Factor; BNT: Boston Naming Test; BVMT-R: Brief Visuospatial Memory Test-Revised; CDR: Clinical Dementia Rating; CEN: Central Executive Network; CVLTII: California Verbal Learning Test, second edition; DLPFC: Dorsolateral Prefrontal Cortex; DMN: Default Mode Network; DSMB: Data Safety Monitoring Board; DSM-IV: Fourth Edition of Diagnostic and Statistical Manual of Mental Disorders; ECog: Everyday Cognition Scale; FAQ: Functional Assessment Questionnaire; FLAIR: Fluid-Attenuated Inversion Recovery; fMRI: Functional Magnetic Resonance Imaging; GDS: Geriatric Depression Scale; ICNs: Intrinsically Connected Networks; IRB: Institutional Review Board; ITI: Inter-Train Interval; ITT: Intention-To-Treat; LPC: Lateral Parietal Cortex; MCl: Mild Cognitive Impairment; MMSE: Mini-Mental State Examination; MNI: Montreal Neurological Institute; MoCA: Montreal Cognitive Assessment; MRI: Magnetic Resonance Imaging; NIBS: Noninvasive Brain Stimulation; $\mathrm{NIH}$ : National Institutes of Health; PCC: Posterior Cingulate Cortex; PE/ NE: Physical/Neurological Examination; rs-fMRI: Resting state functional Magnetic Resonance Imaging; rTMS: Repetitive Transcranial Magnetic Stimulation; SMS: Simultaneous Multi-Slice; SNP: Single Nucleotide Polymorphism; TMS: Transcranial Magnetic Stimulation; VA: Veterans Affairs

\section{Acknowledgements}

We thank our scientific collaborators at Stanford University and medical staff of the VA Palo Alto Health Care System, notably: Jerome A. Yesavage MD, Michael D. Greicius MD MPH and the FIND Lab, Ruth O'Hara PhD and Joachim Hallmayer MD of the MIRECC Translational Core, as well as Steven Chao MD PhD, Jennifer Jennings MD, Makoto Kawai MD, and Logan Schneider MD for their invaluable expertise and assistance on this project. We thank Art Noda MS for his invaluable knowledge and insights on data management and for reviewing a draft of this manuscript.

\section{Sponsor information}

The institutional sponsor is the Palo Alto Veterans Institute for Research (PAVIR), 3801 Miranda Ave, Palo Alto, CA 94304; Telephone 650-858-3970; grants@pavir.org

\section{Authors' contributions}

$J L T, B C H, N D S$ wrote the first draft of this manuscript that describes the study protocol. JLT formulated the research question and wrote the detailed study protocol, along with significant contributions from JWA, JC, and MMA. $J \mathrm{~L}, \mathrm{BCH}, \mathrm{PB}, \mathrm{MWM}$, and NDS participated in the design and optimization of the TMS intervention protocol, including LPC stimulation and the neuronavigation procedure. $\mathrm{BH}$ and $\mathrm{LCL}$ designed and wrote the statistical analyses. NDS, PB, MI, and MWM critically read and revised this protocol manuscript. All authors read and approved the final manuscript.

\section{Funding}

The primary funder is NIH (Award Number R01 AG055526). The NIH Center for Scientific Review organized a scientific peer review of the protocol as part of the grant award process. The current role of the NIH is three-fold: $\mathrm{NIH}$ requires annual progress reports; NIH may determine if an interim analysis should be conducted; $\mathrm{NIH}$ has authority to decide if the study should continue or be stopped. In addition, VA provides institutional support. Specifically, VA Medical Research Service provides trained staff to collect blood samples; VA Sierra-Pacific Mental IIIness Research, Education, and Clinical Center (MIRECC) provides wet laboratory facilities, equipment, and staff to process and store biosamples. Finally, VA Office of Research \& Development provides salary support to M. Windy McNerney through Career Development Award BX004105. The content is solely the responsibility of the authors and does not necessarily represent the official views of $\mathrm{NIH}$ or VA.

\section{Availability of data and materials}

Not applicable.

Data sharing is not relevant to this article. The study was still enrolling study participants at the time of submission; no datasets have been generated or analysed yet

\section{Ethics approval and consent to participate}

The study has been approved by the Stanford University Human Subjects in Medical Research Institutional Review Board (IRB Administrative Panel 3), Palo Alto, CA 94306 (\#FWA00000935) and the National Institutes of Health (NIH) Human Subjects Program in the Office of Extramural Programs. Written informed consent is obtained from study participants at the initial, in-person screening visit $\left(\mathrm{t}_{1}\right)$ by the Study Coordinator and/or Protocol Director. All of the procedures involved, their risks, the potential benefits, and issues of confidentiality, provisions for collection and use of participant data and biological specimens, and provisions for care in the event of harm from trial participation are addressed in the informed consent documents (Additional file 3).

Enough time is allowed for the potential participant to make an informed decision, including time to ask the researchers any questions they may have and discuss the study with others. If the prospective participant lacks adequate decision-making capacity, a designated legal representative will need to sign the consent form, and the participant will need to co-sign to indicate assent. The Research Compliance Officer of the VA Palo Alto Health Care System independently conducts annual audits of the participants' informed consent documents.

\section{Consent for publication}

Not applicable.

\section{Competing interests}

The authors declare that they have no competing interests.

\section{Author details}

${ }^{1}$ US Department of Veterans Affairs (VA) Palo Alto Health Care System (151Y), Sierra-Pacific Mental IIIness Research Education Clinical Center (MIRECC), 3801 Miranda Avenue, Palo Alto, CA 94304-1207, USA. ²Department of Psychiatry and Behavioral Sciences, Stanford University, School of Medicine, Stanford, CA, USA. ${ }^{3}$ War Related IIIness and Injury Study Center (WRIISC), VA Palo Alto Health Care System, Palo Alto, CA, USA. ${ }^{4}$ Department of Radiology, Division of Neuroimaging and Neurointervention, Stanford University Medical Center, Stanford, CA, USA. ${ }^{5}$ Defense and Veterans Brain Injury Center and Polytrauma (DVBIC), VA Palo Alto Health Care System, Palo Alto, CA, USA.

Received: 6 November 2019 Accepted: 3 December 2019 Published online: 16 December 2019

\section{References}

1. Flicker C, Ferris SH, Reisberg B. Mild cognitive impairment in the elderly. Neurology. 1991;41(7):1006.

2. Petersen RC, Smith GE, Waring SC, Ivnik RJ, Tangalos EG, Kokmen E. Mild cognitive impairment: clinical characterization and outcome. Arch Neurol. 1999;56(3):303-8.

3. Petersen RC. Mild cognitive impairment as a diagnostic entity. J Intern Med. 2004;256(3):183-94.

4. Petersen RC, Caracciolo B, Brayne C, Gauthier S, Jelic V, Fratiglioni L. Mild cognitive impairment: a concept in evolution. J Intern Med. 2014;275(3):214-28.

5. Winblad B, Palmer K, Kivipelto M, Jelic V, Fratiglioni L, Wahlund LO, et al. Mild cognitive impairment--beyond controversies, towards a consensus: 
report of the international working group on mild cognitive impairment. Intern Med. 2004;256(3):240-6.

6. Palmer K, Bäckman L, Winblad B, Fratiglioni L. Mild cognitive impairment in the general population: occurrence and progression to Alzheimer disease. Am J Geriatr Psychiatry. 2008;16(7):603-11.

7. Roberts R, Knopman DS. Classification and epidemiology of $\mathrm{MCl}$. Clin Geriatr Med. 2013;29(4):753-72.

8. Mitchell AJ, Shiri-Feshki M. Rate of progression of mild cognitive impairment to dementia--meta-analysis of 41 robust inception cohort studies. Acta Psychiatr Scand. 2009;119(4):252-65

9. Doody RS, Ferris SH, Salloway S, Sun Y, Goldman R, Watkins WE, et al. Donepezil treatment of patients with $\mathrm{MCl}$ : a 48-week randomized, placebocontrolled trial. Neurology. 2009;72(18):1555-61.

10. Petersen RC, Thomas RG, Grundman M, Bennett D, Doody R, Ferris S, et al. Vitamin $\mathrm{E}$ and donepezil for the treatment of mild cognitive impairment. N Engl J Med. 2005;352(23):2379-88.

11. Salloway S, Ferris S, Kluger A, Goldman R, Griesing T, Kumar D, et al. Efficacy of donepezil in mild cognitive impairment: a randomized placebocontrolled trial. Neurology. 2004;63(4):651-7.

12. Cooper C, Li R, Lyketsos C, Livingston G. Treatment for mild cognitive impairment: systematic review. Br J Psychiatry. 2013;203(3):255-64.

13. Fitzpatrick-Lewis D, Warren R, Ali MU, Sherifali D, Raina P. Treatment for mild cognitive impairment: a systematic review and meta-analysis. CMAJ Open. 2015;3(4):E419-27

14. Raschetti R, Albanese E, Vanacore N, Maggini M. Cholinesterase inhibitors in mild cognitive impairment: a systematic review of randomised trials. PLoS Med. 2007;4(11):e338.

15. Tricco AC, Soobiah C, Berliner S, Ho JM, Ng CH, Ashoor HM, et al. Efficacy and safety of cognitive enhancers for patients with mild cognitive impairment: a systematic review and meta-analysis. CMAJ. 2013;185(16):1393-401.

16. Newhouse P, Kellar K, Aisen P, White H, Wesnes K, Coderre E, et al. Nicotine treatment of mild cognitive impairment: a 6-month double-blind pilot clinical trial. Neurology. 2012;78(2):91-101.

17. Rodakowski J, Saghafi E, Butters MA, Skidmore ER. Non-pharmacological interventions for adults with mild cognitive impairment and early stage dementia: An updated scoping review. Mol Aspects Med. 2015;43(44):38-53.

18. Wassermann EM, Zimmermann T. Transcranial magnetic brain stimulation: therapeutic promises and scientific gaps. Pharmacol Ther. 2012;133(1):98-107.

19. Canter RG, Penney J, Tsai LH. The road to restoring neural circuits for the treatment of Alzheimer's disease. Nature. 2016:539(7628):187-96.

20. Drumond Marra HL, Myczkowski ML, Maia Memoria C, Arnaut D, Leite Ribeiro P, Sardinha Mansur CG, et al. Transcranial magnetic stimulation to address mild cognitive impairment in the elderly: a randomized controlled study. Behav Neurol. 2015;2015:287843.

21. Padala PR, Padala KP, Lensing SY, Jackson AN, Hunter CR, Parkes CM, et al. Repetitive transcranial magnetic stimulation for apathy in mild cognitive impairment: a double-blind, randomized, sham-controlled, cross-over pilot study. Psychiatry Res. 2018;261:312-8.

22. Rutherford G, Lithgow B, Moussavi Z. Short and Long-term effects of rTMS treatment on Alzheimer's disease at different stages: a pilot study. J Exp Neurosci. 2015;9:43-51.

23. Ahmed MA, Darwish ES, Khedr EM, El Serogy YM, Ali AM. Effects of low versus high frequencies of repetitive transcranial magnetic stimulation on cognitive function and cortical excitability in Alzheimer's dementia. J Neurol. 2012;259(1):83-92.

24. Rabey JM, Dobronevsky E, Aichenbaum S, Gonen O, Marton RG, Khaigrekht M. Repetitive transcranial magnetic stimulation combined with cognitive training is a safe and effective modality for the treatment of Alzheimer's disease: a randomized, double-blind study. J Neural Transm Vienna. 2013;120(5):813-9.

25. Cotelli M, Calabria M, Manenti R, Rosini S, Zanetti O, Cappa SF, et al. Improved language performance in Alzheimer disease following brain stimulation. J Neurol Neurosurg Psychiatry. 2011:82(7):794-7.

26. Cotelli M, Manenti R, Cappa SF, Zanetti O, Miniussi C. Transcranial magnetic stimulation improves naming in Alzheimer disease patients at different stages of cognitive decline. Eur J Neurol. 2008;15(12):1286-92.

27. Cotelli M, Manenti R, Cappa SF, Geroldi C, Zanetti O, Rossini PM, et al. Effect of transcranial magnetic stimulation on action naming in patients with Alzheimer disease. Arch Neurol. 2006;63(11):1602-4.

28. Maeda F, Keenan JP, Tormos JM, Topka H, Pascual-Leone A. Interindividual variability of the modulatory effects of repetitive transcranial magnetic stimulation on cortical excitability. Exp Brain Res. 2000;133(4):425-30.
29. Koch G, Bonni S, Pellicciari MC, Casula EP, Mancini M, Esposito R, et al. Transcranial magnetic stimulation of the precuneus enhances memory and neural activity in prodromal Alzheimer's disease. Neuroimage. 2018;169:302-11.

30. Dubois B, Hampel H, Feldman HH, Scheltens P, Aisen P. Andrieu S, et al. Preclinical Alzheimer's disease: definition, natural history, and diagnostic criteria. Alzheimer's \& dementia : the journal of the Alzheimer's Association. 2016;12(3):292-323.

31. Turriziani P, Smirni D, Zappala G, Mangano GR, Oliveri M, Cipolotti L. Enhancing memory performance with rTMS in healthy subjects and individuals with mild cognitive impairment: the role of the right dorsolateral prefrontal cortex. Front Hum Neurosci. 2012;6:62.

32. Solé-Padullés C, Bartrés-Faz D, Junqué C, Clemente IC, Molinuevo JL, Bargalló N, et al. Repetitive Transcranial magnetic stimulation effects on brain function and cognition among elders with memory dysfunction. A Randomized Sham-Controlled Study Cerebral Cortex. 2006;16(10):1487-93.

33. Chen R, Classen J, Gerloff C, Celnik P, Wassermann EM, Hallett M, et al. Depression of motor cortex excitability by low-frequency transcranial magnetic stimulation. Neurology. 1997;48(5):1398-403.

34. Lee J, Choi BH, Oh E, Sohn EH, Lee AY. Treatment of Alzheimer's disease with repetitive Transcranial magnetic stimulation combined with cognitive training: a prospective, randomized, double-blind. Placebo-Controlled Study J Clin Neurol. 2016;12(1):57-64.

35. Wu Y, Xu W, Liu X, Xu Q, Tang L, Wu S. Adjunctive treatment with high frequency repetitive transcranial magnetic stimulation for the behavioral and psychological symptoms of patients with Alzheimer's disease: a randomized, double-blind, sham-controlled study. Shanghai Arch Psychiatry. 2015;27(5):280-8.

36. Zhao J, Li Z, Cong Y, Zhang J, Tan M, Zhang H, et al. Repetitive transcranial magnetic stimulation improves cognitive function of Alzheimer's disease patients. Oncotarget. 2017;8(20):33864-71.

37. Heath A, Taylor JL, McNerney MW. rTMS for the treatment of Alzheimer's disease: where should we be stimulating? Expert Rev Neurother. 2018; 18(12):903-5.

38. Wang JX, Rogers LM, Gross EZ, Ryals AJ, Dokucu ME, Brandstatt KL, et al Targeted enhancement of cortical-hippocampal brain networks and associative memory. Science. 2014;345(6200):1054-7.

39. Badhwar A, Tam A, Dansereau C, Orban P, Hoffstaedter F, Bellec P. Restingstate network dysfunction in Alzheimer's disease: a systematic review and meta-analysis. Alzheimer's \& Dementia: Diagnosis, Assessment \& Disease Monitoring. 2017:8:73-85.

40. Wang K, Liang M, Wang L, Tian L, Zhang X, Li K, et al. Altered functional connectivity in early Alzheimer's disease: a resting-state fMRI study. Hum Brain Mapp. 2007;28(10):967-78.

41. Alcala-Lozano R, Morelos-Santana E, Cortes-Sotres JF, Garza-Villarreal EA, Sosa-Ortiz AL, Gonzalez-Olvera JJ. Similar clinical improvement and maintenance after rTMS at $5 \mathrm{~Hz}$ using a simple vs. complex protocol in Alzheimer's disease. Brain stimulation. 2018;11(3):625-7.

42. Weiner M, Petersen R. Alzheimer's Disease Neuroimaging Initiative 3 (ADNI3) Protocol. Retrieved from http://adni.loni.usc.edu/wp-content/themes/ freshnews-dev-v2/documents/clinical/ADNI3_Protocol.pdf 2016.

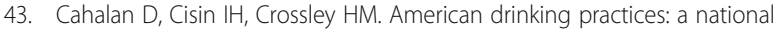
survey of behavior and attitudes. NJ: New Brunswick; 1969.

44. Liao X, Li G, Wang A, Liu T, Feng S, Guo Z, et al. Repetitive transcranial magnetic stimulation as an alternative therapy for cognitive impairment in Alzheimer's disease: a meta-analysis. J Alzheimers Dis. 2015;48:463-72.

45. Hsu WY, Ku Y, Zanto TP, Gazzaley A. Effects of noninvasive brain stimulation on cognitive function in healthy aging and Alzheimer's disease: a systematic review and meta-analysis. Neurobiol Aging. 2015;36(8):2348-59.

46. Fox MD, Buckner RL, White MP, Greicius MD, Pascual-Leone A. Efficacy of transcranial magnetic stimulation targets for depression is related to intrinsic functional connectivity with the subgenual cingulate. Biol Psychiatry. 2012;72(7):595-603.

47. Mi Z, Biswas K, Fairchild JK, Davis-Karim A, Phibbs CS, Forman SD, et al. Repetitive transcranial magnetic stimulation rTMS for treatment-resistant major depression (TRMD) veteran patients: study protocol for a randomized controlled trial. Trials. 2017;18(1):409.

48. Wechsler D. Manual for the Wechsler memory scale-revised. San Antonio, TX: The Psychological Corporation; 1987.

49. Folstein MF, Folstein SE, McHugh PR. "mini-mental state". A practical method for grading the cognitive state of patients for the clinician. J Psychiatr Res. 1975;12(3):189-98. 
50. Morris JC. The clinical dementia rating (CDR): current version and scoring rules. Neurology. 1993;43(11):2412-4.

51. Yesavage JA, Brink TL, Rose TL. Development and validation of a geriatric depression screening scale: a preliminary report. J Psychiatr Res. 1982;17:37-49.

52. Rattanabannakit C, Risacher SL, Gao S, Lane KA, Brown SA, McDonald BC, et al. The cognitive change index as a measure of self and informant perception of cognitive decline: relation to neuropsychological tests. J Alzheimers Dis. 2016;51(4):1145-55.

53. Rosen WG, Terry RD, Fuld PA, Katzman R, Peck A. Pathological verification of ischemic score in differentiation of dementias. Ann Neurol. 1980;7(5):486-8.

54. Delis DC, Kramer JH, Kaplan E, Ober B. The California verbal learning testsecond edition. New York: Psychological Corporation; 2000.

55. Karrasch M, Sinerva E, Gronholm P, Rinne J, Laine M. CERAD test performances in amnestic mild cognitive impairment and Alzheimer's disease. Acta Neurol Scand. 2005:111(3):172-9.

56. Ellis KA, Bush Al, Darby D, De Fazio D, Foster J, Hudson P, et al. The Australian imaging, biomarkers and lifestyle (AIBL) study of aging: methodology and baseline characteristics of 1112 individuals recruited for a longitudinal study of Alzheimer's disease. Int Psychogeriatr. 2009;21(4):672-87.

57. Ribeiro F, de Mendonca A, Guerreiro M. Mild cognitive impairment: deficits in cognitive domains other than memory. Dement Geriatr Cogn Disord. 2006;21(5-6):284-90

58. Silva D, Guerreiro M, Maroco J, Santana I, Rodrigues A, Bravo Marques J, et al. Comparison of four verbal memory tests for the diagnosis and predictive value of mild cognitive impairment. Dement Geriatr Cogn Dis Extra. 2012;2:120-31.

59. Rabin LA, Pare N, Saykin AJ, Brown MJ, Wishart HA, Flashman LA, et al, Differential memory test sensitivity for diagnosing amnestic mild cognitive impairment and predicting conversion to Alzheimer's disease. Neuropsychol Dev Cogn B Aging Neuropsychol Cogn. 2009;16(3):357-76.

60. Long NM, Oztekin I, Badre D. Separable prefrontal cortex contributions to free recall. J Neurosci. 2010;30(33):10967-76.

61. Bentwich J, Dobronevsky E, Aichenbaum S, Shorer R, Peretz R, Khaigrekht M, et al. Beneficial effect of repetitive transcranial magnetic stimulation combined with cognitive training for the treatment of Alzheimer's disease: a proof of concept study. J Neural Transm Vienna. 2011;118(3):463-71.

62. Devi G, Voss HU, Levine D, Abrassart D, Heier L, Halper J, et al. Open-label, short-term, repetitive transcranial magnetic stimulation in patients with Alzheimer's disease with functional imaging correlates and literature review. Am J Alzheimers Dis Other Dement. 2014;29(3):248-55.

63. Nasreddine ZS, Phillips NA, Bedirian V, Charbonneau S, Whitehead V, Collin I, et al. The Montreal cognitive assessment, MoCA: a brief screening tool for mild cognitive impairment. J Am Geriatr Soc. 2005;53(4):695-9.

64. Benedict RHB, Schretlen D, Groninger L, Dobraski M, Sphritz B. Revision of the brief Visuospatial memory test: studies of normal performance, reliability and validity. Psychol Assess. 1996;8:145-53.

65. Morris JC, Heyman A, Mohs RC, Hughes JP, van Belle G, Fillenbaum G, et al. The consortium to establish a registry for Alzheimer's disease (CERAD). Part I. clinical and neuropsychological assessment of Alzheimer's disease. Neurology. 1989:39(9):1159-65.

66. Huff FJ, Collins C, Corkin S, Rosen TJ. Equivalent forms of the Boston naming test. J Clin Exp Neuropsychol. 1986;8(5):556-62.

67. Hubley AM. Using the Rey-Osterrieth and modified Taylor complex figures with older adults: a preliminary examination of accuracy score comparability. Arch Clin Neuropsychol. 2010;25(3):197-203.

68. Hubley AM, Jassal S. Comparability of the Rey-Osterrieth and modified Taylor complex figures using total scores, completion times, and construct validation. J Clin Exp Neuropsychol. 2006:28(8):1482-97.

69. Partington JE, Leiter RG. Partington's pathway test. The Psychological Service Center Bulletin. 1949:1:9-20.

70. Reitan RM. Validity of the trail-making test as an indication of organic brain damage. Perceptual Motor Skills. 1958;8:271-6.

71. Farias ST, Mungas D, Reed BR, Cahn-Weiner D, Jagust W, Baynes K, et al. The measurement of everyday cognition (ECog): scale development and psychometric properties. Neuropsychology. 2008;22(4):531-44.

72. Pfeffer Rl, Kurosaki TT, Harrah CH Jr, Chance JM, Filos S. Measurement of functional activities in older adults in the community. J Gerontol. 1982;37(3):323-9.

73. Singer JD, Willett JB. Applied longitudinal data analysis: modeling change and event Occurence. New York: Oxford University Press; 2003.

74. Dosenbach NUF, Fair DA, Cohen AL, Schlaggar BL, Petersen SE. A dual-networks architecture of top-down control. Trends Cogn Sci. 2008;12(3):99-105.
75. Touroutoglou A, Hollenbeck M, Dickerson BC, Feldman BL. Dissociable large-scale networks anchored in the right anterior insula subserve affective experience and attention. Neuroimage. 2012;60(4):1947-58.

76. Brier MR, Thomas JB, Snyder AZ, Benzinger TL, Zhang D, Raichle ME, et al. Loss of intranetwork and internetwork resting state functional connections with Alzheimer's disease progression. J Neurosci. 2012;32(26):8890-9.

77. Dayan E, Censor N, Buch ER, Sandrini M, Cohen LG. Noninvasive brain stimulation: from physiology to network dynamics and back. Nat Neurosci. 2013;16(7):838-44.

78. Ruff CC, Driver J, Bestmann S. Combining TMS and fMRI: from 'virtual lesions' to functional-network accounts of cognition. Cortex. 2009;45(9):1043-9.

79. Buckner RL, Andrews-Hanna JR, Schacter DL. The brain's default network: anatomy, function, and relevance to disease. Ann N Y Acad Sci. 2008;1124:1-38.

80. Gusnard DA, Akbudak E, Shulman GL, Raichle ME. Medial prefrontal cortex and self-referential mental activity: relation to a default mode of brain function. Proc Natl Acad Sci U S A. 2001;98(7):4259-64.

81. Shulman GL, Fiez JA, Corbetta M, Buckner RL, Miezin FM, Raichle ME, et al. Common blood flow changes across visual tasks: II. Decreases in cerebral cortex. J Cogn Neurosci. 1997;9(5):648-63.

82. Vincent JL, Kahn I, Snyder AZ, Raichle ME, Buckner RL. Evidence for a frontoparietal control system revealed by intrinsic functional connectivity. J Neurophysiol. 2008;100(6):3328-42.

83. Chen AC, Oathes DJ, Chang C, Bradley T, Zhou ZW, Williams LM, et al. Causal interactions between fronto-parietal central executive and defaultmode networks in humans. Proc Natl Acad Sci U S A. 2013;110(49):19944-9.

84. Wang JX, Voss JL. Long-lasting enhancements of memory and hippocampal-cortical functional connectivity following multiple-day targeted noninvasive stimulation. Hippocampus. 2015;25(8):877-83.

85. Fox MD, Buckner RL, Liu H, Chakravarty MM, Lozano AM, Pascual-Leone A. Resting-state networks link invasive and noninvasive brain stimulation across diverse psychiatric and neurological diseases. Proc Natl Acad Sci U S A. 2014;111(41):E4367-75.

86. Lau WKW, Leung MK, Lee TMC, Law ACK. Resting-state abnormalities in amnestic mild cognitive impairment: a meta-analysis. Transl Psychiatry. 2016;6:e790.

87. Greicius MD, Supekar K, Menon V, Dougherty RF. Resting-state functional connectivity reflects structural connectivity in the default mode network. Cereb Cortex. 2009;19(1):72-8.

88. Leech R, Kamourieh S, Beckmann CF, Sharp DJ. Fractionating the default mode network: distinct contributions of the ventral and dorsal posterior cingulate cortex to cognitive control. J Neurosci. 2011;31(9):3217-24.

89. Margulies DS, Vincent JL, Kelly C, Lohmann G, Uddin LQ, Biswal BB, et al. Precuneus shares intrinsic functional architecture in humans and monkeys. Proc Natl Acad Sci U S A. 2009;106(47):20069-74.

90. Spreng RN, Schacter DL. Default network modulation and large-scale network interactivity in healthy young and old adults. Cereb Cortex. 2012;22(11):2610-21.

91. Liston C, Chen AC, Zebley BD, Drysdale AT, Gordon R, Leuchter B, et al. Default mode network mechanisms of transcranial magnetic stimulation in depression. Biol Psychiatry. 2014;76(7):517-26.

92. Grady C, Sarraf S, Saverino C, Campbell K. Age differences in the functional interactions among the default, frontoparietal control, and dorsal attention networks. Neurobiol Aging. 2016;41:159-72.

93. Ng KK, Lo JC, Lim JKW, Chee MWL, Zhou J. Reduced functional segregation between the default mode network and the executive control network in healthy older adults: a longitudinal study. Neuroimage. 2016;133:321-30.

94. Wang $P$, Zhou B, Yao H, Zhan $Y$, Zhang Z, Cui $Y$, et al. Aberrant intra- and inter-network connectivity architectures in Alzheimer's disease and mild cognitive impairment. Sci Rep. 2015;5:14824.

95. Jack CR, Bennett DA, Blennow K, Carrillo MC, Dunn B, Haeberlein SB, et al. NIA-AA research framework: toward a biological definition of Alzheimer's disease. Alzheimers Dement. 2018;14(4):535-62.

96. LaFerla FM, Green KN, Oddo S. Intracellular amyloid-beta in Alzheimer's disease. Nat Rev Neurosci. 2007:8(7):499-509.

97. Nisbet RM, Polanco JC, Ittner LM, Gotz J. Tau aggregation and its interplay with amyloid-beta. Acta Neuropathol. 2015;129(2):207-20.

98. Kaufman SK, Sanders DW, Thomas TL, Ruchinskas AJ, Vaquer-Alicea J, Sharma AM, et al. Tau prion strains dictate patterns of cell pathology, progression rate, and regional vulnerability in vivo. Neuron. 2016;92(4):796-812.

\section{Publisher's Note}

Springer Nature remains neutral with regard to jurisdictional claims in published maps and institutional affiliations. 